\title{
Nilpotence relations in products of groups
}

\author{
Giulio Francalanci* \\ Communicated by Timothy C. Burness \\ This work is dedicated to Carlo Casolo. We will always remember \\ your brilliance, your knowledge and your humanity.
}

\begin{abstract}
Two subgroups $A$ and $B$ of a group $G$ are said to be $\mathcal{N}$-connected if, for all $a$ in $A$ and $b$ in $B$, the subgroup generated by $a$ and $b$ is a nilpotent group. In this paper, we study the structure of a group $G$ assuming that $G=A B$ and $A$ and $B$ are $\mathcal{N}$-connected subgroups satisfying Max or Min.
\end{abstract}

\section{Introduction}

Let $G$ be a group and $A$ and $B$ two subgroups; $G$ is said to be the product of $A$ and $B$ if $G=\{a b \mid a \in A, b \in B\} . G$ is said to be factorized and $A$ and $B$ are said to be factors of $G$.

This definition gives rise to the following general structure problem.

Problem. Let $G$ be a factorized group, $G=A B$, with $A$ and $B$ subgroups, and assume that $A$ and $B$ satisfy some group-theoretical property $\mathcal{P}$. What can be said about the structure of the whole group $G$ ?

A well-known statement of this type is, for instance, the theorem of N. Itô [4] which says that if the group $G$ is the product of two abelian subgroups, then $G$ is metabelian. Other examples are the papers of O. Kegel [5] and H. Wielandt [10] where it has been proved that the product $G$ of two finite nilpotent subgroups $A$ and $B$ is solvable.

More results have been obtained under additional assumptions such as, for instance, the (generalized) solvability of the group $G$.

Let $C$ be a non-empty class of groups; let $G$ be a group, and consider $A$ and $B$, two subgroups of $G$. We say that the subgroups $A$ and $B$ are $C$-connected if, for all $a \in A$ and $b \in B$, the subgroup $\langle a, b\rangle$ belongs to $e$. This definition was

This work has been partially funded by Gruppo Nazionale per le Strutture Algebriche, Geometriche e le loro Applicazioni (GNSAGA) of the Istituto Nazionale di Alta Matematica "Francesco Severi" (INDAM). 
formulated for the first time by A. Carocca in [3] where he studied the product of $\zeta$-connected finite groups when $\mathcal{C}$ is the class of nilpotent groups. In this paper, we prove two theorems, without the assumption of finiteness. We use $\mathcal{N}$ to indicate the class of nilpotent groups.

Theorem A. Let $G$ be a group and $A, B \leq G=A B$, where $A$ and $B$ are supersolvable $\mathcal{N}$-connected subgroups. Then $G$ is supersolvable.

Theorem A has the following corollaries.

Corollary 1.1. Let $G$ be a group and $A, B \leq G=A B$, where $A$ and $B$ are finitely generated nilpotent $\mathcal{N}$-connected subgroups. Then $G$ is a finitely generated nilpotent group.

Corollary 1.2. Let $G$ be a group, $G=G_{1} G_{2} \ldots G_{n}$, where $G_{1}, G_{2}, \ldots, G_{n}$ are pairwise permutable supersolvable (finitely generated nilpotent) subgroups of $G$. If, for all $i, j: 1, \ldots, n$ such that $i \neq j$, we have that $G_{i}$ and $G_{j}$ are $\mathcal{N}$-connected, then $G$ is supersolvable (finitely generated nilpotent).

The second theorem is the following.

Theorem B. Let $G$ be a group, and let $A, B$ be two subgroups of $G$ such that $G=A B$. Assume that $A$ and $B$ are $\mathcal{N}$-connected and Černikov groups. Then $G$ is a Černikov group.

Also, in this case, we may deduce two corollaries.

Corollary 1.3. Let $G$ be a group, and let $A, B$ be two subgroups of $G$ such that $G=A B$. Assume that $A$ and $B$ are $\mathcal{N}$-connected solvable groups with Min. Then $G$ is a solvable group with Min.

Corollary 1.4. Let $G$ be a group, $G=G_{1} G_{2} \ldots G_{n}$, where $G_{1}, G_{2}, \ldots, G_{n}$ are pairwise permutable Černikov (solvable with Min) subgroups of $G$. If, for all $i, j: 1, \ldots, n$ such that $i \neq j$, we have that $G_{i}$ and $G_{j}$ are $\mathcal{N}$-connected, then $G$ is Černikov (solvable with Min).

\section{Preliminaries}

In this section, we collect the main definitions and results we need in the proofs of the theorems. 
Lemma 2.1 ([2,10]). Let the group $G=A B$ be the product of two subgroups $A$ and $B$. For a subgroup $S$ of $G$, the following conditions are equivalent:

(1) if $a b \in S$, with $a \in A$ and $b \in B$, then $a \in S$;

(2) $S=(A \cap S)(B \cap S)$ and $A \cap B \leq S$.

Definition 1 ([2,10]). A subgroup $S$ of a factorized group $G$ is said to be factorized if it satisfies one of the equivalent conditions of Lemma 2.1.

Lemma 2.2. Let the group $G=A B$ be factorized by $A, B \leq G$. Let the group $D$ be such that $B \leq D \leq G$, and let a subgroup $Y \leq G$ be factorized in $A D$. Then it is also factorized in $A B$.

Proof. By Definition 1, we have $Y=(A \cap Y)(D \cap Y)$ and $Y \geq A \cap D$. Applying the Dedekind modular law twice, we get

$$
D \cap Y=A B \cap D \cap Y=(A \cap D) B \cap Y=(A \cap D)(B \cap Y)
$$

and therefore

$$
Y=(A \cap Y)(D \cap Y)=(A \cap Y)(A \cap D)(B \cap Y)=(A \cap Y)(B \cap Y)
$$

Definition 2 ([8, Chapter 3, p. 64]). A group $G$ is said to satisfy Max, the maximal condition on subgroups, if, for all chains $H_{0} \leq H_{1} \leq H_{2} \leq \cdots$, there exists $n \in \mathbb{N}$ such that $H_{n}=H_{n+1}=\cdots$ or, in other words, every ascending chain of subgroups is finite. Analogously, a group $G$ is said to satisfy Min, the minimal condition on subgroups, if, for all chains $H_{0} \geq H_{1} \geq H_{2} \geq \cdots$, there exists $n \in \mathbb{N}$ such that $H_{n}=H_{n+1}=\cdots$ or, in other words, every descending chain of subgroups is finite.

In the rest of this paper, we deal with some classes of groups satisfying Max or Min. For this reason, we recall them.

Definition 3 ([8, Chapter 5, p. 147]). A group is said to be polycyclic if it has a finite cyclic series.

Polycyclic groups are characterized as follows.

Proposition 2.3 ([8, Chapter 5, Proposition 5.4.12, p. 147]). A group is polycyclic if and only if it is solvable and satisfies the maximal condition on subgroups.

Important subclasses of polycyclic groups are finitely generated nilpotent groups and supersolvable groups. 
Definition 4 ([8, Chapter 5, p. 145]). A group is said to be supersolvable if it has a finite normal cyclic series.

We summarize the main properties of those groups.

Proposition 2.4 ([8,9]). Let $G$ be a polycyclic group. Then

(1) $G$ is nilpotent if and only if every finite quotient of $G$ is nilpotent,

(2) $G$ is supersolvable if and only if every finite quotient of $G$ is supersolvable;

moreover, if $G$ is supersolvable, then

(3) $\operatorname{Fit}(G)$ is nilpotent and $G / \operatorname{Fit}(G)$ is finite abelian,

(4) $1 \neq N \triangleleft G$ implies $1 \neq\langle x\rangle \triangleleft G$, for some $x \in N$.

A fundamental tool for our investigation is the following invariant.

Definition 5 ([8, Chapter 5, Proposition 5.4.13, p. 147]). Let $G$ be a polycyclic group. The number of infinite factors in a cyclic series is independent of the series; thus it is an invariant, called the Hirsch length of $G, h(G)$.

An important class of groups that satisfies the minimal conditions on subgroups is the class of Černikov groups.

Definition 6 ([8, Chapter 5, p. 151]). A group is said to be Černikov if it is an extension of a finite direct product of quasicyclic groups by a finite group.

The following characterization holds.

Proposition 2.5 ([8, Chapter 5, Proposition 5.4.23, p. 151]). A solvable group satisfies the minimal condition on subgroups if and only if it is a solvable Černikov group.

A class of groups that generalizes both polycyclic and Černikov groups is the class of minimax groups.

Definition 7 ([6, Chapter 5, p. 86]). A group is said to be minimax if it has a series of finite length for which each factor satisfies Max or Min.

We introduced this class of groups to define the following invariant. 
Definition 8 ([6, Chapter 5, p. 87]). Let $G$ be a solvable-by-finite minimax group. We know that there exists a series $1=G_{0} \triangleleft G_{1} \triangleleft \cdots \triangleleft G_{n}=G$ in which each factor satisfies Max or Min. Clearly, it is possible to refine this series and obtain a series with cyclic factors (finite or infinite) and quasicyclic factors. By a routine application of the Schreier refinement theorem, it is possible to show that the number of infinite factors of that series is an invariant, and we call it the minimax length or minimality of $G, m(G)$. It can be thought of as a generalization of Hirsch length for polycyclic groups.

To end this section, we give the definition of formation and saturated formation, and we state the theorem of Carocca.

Definition 9 ([8, Chapter 9, p. 269]). A class of finite groups $\mathcal{F}$ is said to be a formation if

(1) every homomorphic image of an $\mathcal{F}$-group is an $\mathcal{F}$-group,

(2) if $\frac{G}{N_{1}}, \frac{G}{N_{2}} \in \mathcal{F}$, then $\frac{G}{N_{1} \cap N_{2}} \in \mathcal{F}$.

Moreover, a formation $\mathcal{F}$ is said to be saturated if $G \in \mathcal{F}$ whenever $\frac{F}{\Phi(G)} \in \mathcal{F}$

Theorem 2.6 ([3]). Let $G=G_{1} G_{2} \ldots G_{r}$ be a finite group such that $G_{1}, \ldots, G_{r}$ are pairwise permutable subgroups of $G$. Let $\mathcal{F}$ be a saturated formation such that $\mathcal{N} \subseteq \mathcal{F}$. If, for every pair $i, j \in\{1, \ldots, r\}, i \neq j$, the subgroups $G_{i}$ and $G_{j}$ are $\mathcal{N}$-connected $\mathcal{F}$-groups, then $G \in \mathcal{F}$.

\section{Proof of Theorem A}

Lemma 3.1. Let $G$ be a group, and let $A, B \leq G$ be such that $G=A B$ and $A, B$ are $\mathcal{N}$-connected. If $A$ and $B$ are supersolvable (f.g. nilpotent), then $G$ is polycyclic if and only if it is supersolvable (f.g. nilpotent).

Proof. If $G$ is supersolvable (f.g. nilpotent), the result is clear. Suppose that $G$ is polycyclic and $A$ and $B$ are supersolvable. Consider a normal subgroup $N$ of finite index in $G$. We have

$$
\frac{G}{N}=\frac{A N}{N} \frac{B N}{N},
$$

that is, a product of finite supersolvable $\mathcal{N}$-connected subgroups. The class of finite supersolvable groups is a saturated formation that contains the class of finite nilpotent groups. So we can apply Carocca's theorem, Theorem 2.6, and we get that $G / N$ is supersolvable. Thus, we have a polycyclic group in which every finite 
homomorphic image is supersolvable. Applying Proposition 2.4(2), $G$ is supersolvable. The same holds when $A$ and $B$ are finitely generated nilpotent subgroups, using, in this case, Proposition 2.4 (1).

Before stating the main theorem of this section, we need to point out some technical properties.

Lemma 3.2. Let $G$ be a group and $H, K \leq G$ such that $G=\langle H, K\rangle$. If we have $|G: K|<\infty$, then $|H: H \cap K|<\infty$

Lemma 3.3. Let $G$ be a finitely generated nilpotent group and $H \leq G$ such that $N_{G}(H) / H$ is a finite group. Then $|G: H|<\infty$

Corollary 3.4. Let $G$ be a supersolvable group, $F=\operatorname{Fit}(G)$ and $H \leq F$ such that $N_{G}(H) / H$ is a finite group. Then $|G: H|<\infty$

Proof. Consider $G$ and $H$ as in the hypotheses. Clearly, $\left|N_{F}(H): H\right|<\infty$. Applying Lemma 3.3, $|F: H|<\infty$, and together with the fact that $|G: F|<\infty$ by Proposition 2.4, we have the thesis.

Lemma 3.5. Let $G$ be a group, $A, B \leq G$ such that $G=A B$. If $A$ satisfies Max, then $G$ satisfies the maximal condition on the subgroups containing $B$.

Proof. Consider $S_{1} \leq S_{2} \leq \cdots$ an ascending chain of subgroups containing $B$. Each $S_{i}$ is factorized, with the following factorization:

$$
S_{i}=\left(S_{i} \cap A\right) B .
$$

The chain $S_{1} \cap A \leq S_{2} \cap A \leq \cdots$ is an ascending chain in $A$, which is a group that satisfies Max. Thus, there exists $t \in \mathbb{N}$ such that $S_{t}=S_{t+j}$ for all $j \geq 0$. Hence,

$$
S_{t}=\left(S_{t} \cap A\right) B=\left(S_{t+j} \cap A\right) B=S_{t+j},
$$

and the thesis follows.

Proof of Theorem A. We proceed by induction on $h:=h(A)+h(B)$, where $h(X)$ is the Hirsch length of the group $X$. If $h(A)=h(B)=0$, then $A$ and $B$ are finite, and the result holds by Theorem 2.6. If $h(A)=0$, then $A$ is finite, so $B$ is a subgroup of finite index in $G$; hence, since $B_{G}$ is a normal supersolvable subgroup and the factor $G / B_{G}$ is a supersolvable finite group, we conclude using Lemma 3.1. Thus, we can assume that both $h(A), h(B) \geq 1$. We define the sets

$$
\begin{aligned}
& \mathcal{A}=\{S \mid A \leq S \leq G, S \text { supersolvable, } h(S \cap B) \geq 1\}, \\
& \mathcal{B}=\{T \mid B \leq T \leq G, T \text { supersolvable, } h(T \cap A) \geq 1\} .
\end{aligned}
$$


We prove that either $\mathcal{A}$ or $\mathscr{B}$ is non-empty. In fact, if $h(A \cap B) \geq 1$, both $\mathcal{A}$ and $\mathscr{B}$ are non-empty. Suppose that $h(A \cap B)=0$, i.e. $A \cap B$ is a finite group; by Proposition 2.4, we can choose $a \in A, b \in B$ such that $|a|=\infty=|b|$ and $\langle a\rangle \triangleleft A$, $\langle b\rangle \triangleleft B$. By $\mathcal{N}$-connection, we know that $\langle a, b\rangle$ is an infinite nilpotent group, and for this reason, we have that

$$
Z(\langle a, b\rangle) \leq C_{G}(a) \cap C_{G}(b) \leq N_{G}(\langle a\rangle) \cap N_{G}(\langle b\rangle) .
$$

It is then easy to prove that at least one of $N_{A}(\langle b\rangle)$ and $N_{B}(\langle a\rangle)$ is infinite. Suppose that $N_{A}(\langle b\rangle)$ is infinite, and let $T=N_{G}(\langle b\rangle)$.

If $\left|A: N_{A}(\langle b\rangle)\right|=\infty$, then observing that

$$
T=N_{A}(\langle b\rangle) B \quad \text { and } \quad h\left(N_{A}(\langle b\rangle)\right)<h(A),
$$

we get that $T$ is supersolvable by the inductive hypothesis, and $\mathscr{B} \neq \emptyset$. Otherwise, if $\left|A: N_{A}(\langle b\rangle)\right|<\infty$, consider

$$
\frac{T}{\langle b\rangle}=\frac{N_{A}(\langle b\rangle)\langle b\rangle}{\langle b\rangle} \frac{B}{\langle b\rangle} .
$$

Observing that $h(\langle b\rangle)=1$, by the inductive hypothesis we have that $T /\langle b\rangle$ is supersolvable, and by Lemma 3.1, $T$ is supersolvable. Hence, $\mathscr{B} \neq \varnothing$.

Clearly, by Lemma $3.5, \mathscr{B}$ admits a maximal element $D$. Stressing that every subgroup containing $A$ or $B$ factorizes by Lemma 2.1 , we get $D=(D \cap A) B$.

We know by the definition of Hirsch length that $h(A \cap D) \leq h(A)$. Suppose by contradiction that $h(A \cap D)<h(A)$, i.e. $|A: D \cap A|=\infty$. Set $F_{A}=\operatorname{Fit}(A)$ and $F_{D}=\operatorname{Fit}(D)$.

Define the following chain of subgroups:

$$
M_{0}=F_{A} \cap F_{D}, \quad M_{1}=N_{F_{D}}\left(M_{0}\right), \quad \ldots, \quad M_{i}=N_{F_{D}}\left(M_{i-1}\right) .
$$

We know that $F_{D}$ is nilpotent and normal in $D$, so there exists $t \in \mathbb{N}$ such that $M_{t}=N_{F_{D}}\left(M_{t-1}\right)=F_{D}$. Thus, we show by induction on $i$ that the following factorization holds for all $i: 1 \ldots t$ :

$$
N_{G}\left(M_{i}\right)=N_{A}\left(M_{i}\right) N_{D}\left(M_{i}\right) .
$$

If $i=0$, consider $g \in N_{G}\left(M_{0}\right)$; then $g=a b$ for certain $a \in A$ and $b \in D$, and from $M_{0}^{g}=M_{0}$, we deduce $M_{0}^{a}=M_{0}^{b^{-1}} \leq F_{A} \cap F_{D}=M_{0}$. Knowing that $M_{0}$ is nilpotent, we get $a \in N_{A}\left(M_{0}\right)$, which is the thesis.

Suppose that $i \geq 1$, and consider $g \in N_{G}\left(M_{i}\right)$. As before, $g=a b$ with $a \in A$, $b \in D$, and then $M_{i}^{a}=M_{i}^{b^{-1}}$. It is clear that

$$
M_{0}^{a} \leq F_{A} \cap M_{i}^{a}=F_{A} \cap M_{i}^{b^{-1}} \leq F_{A} \cap F_{D},
$$


that is, $M_{0}^{a} \leq M_{0}$, i.e. $a \in N_{A}\left(M_{0}\right)$. Suppose that $0 \leq j \leq i$ is the greatest index such that $a \in N_{A}\left(M_{j}\right)$, and suppose that $j \leq i-1$. Knowing that

$$
M_{j+1}^{a} \leq M_{i}^{a}=M_{i}^{b^{-1}} \leq F_{D}
$$

and $\left\langle a, M_{j+1}\right\rangle \leq N_{G}\left(M_{j}\right)$, we deduce

$$
M_{j+1}^{a} \leq N_{G}\left(M_{j}\right) \cap F_{D}=N_{F_{D}}\left(M_{j}\right)=M_{j+1}
$$

that is, $M_{j+1}^{a} \leq M_{j+1}$. By inductive hypothesis, $N_{G}\left(M_{j}\right)=N_{A}\left(M_{j}\right) N_{D}\left(M_{j}\right)$, and by Lemma 2.2, $N_{G}\left(M_{j}\right)=N_{A}\left(M_{j}\right) N_{B}\left(M_{j}\right)$. Passing to the quotient,

$$
\frac{N_{G}\left(M_{j}\right)}{M_{j}}=\frac{N_{A}\left(M_{j}\right) M_{j}}{M_{j}} \frac{N_{B}\left(M_{j}\right) M_{j}}{M_{j}} .
$$

These two factors are $\mathcal{N}$-connected supersolvable, and using the properties of Hirsch length and the fact that $h\left(M_{j}\right) \geq h\left(M_{0}\right) \geq 1$, we can say that $N_{G}\left(M_{j}\right) / M_{j}$ is supersolvable, and hence, by Lemma 3.1, $N_{G}\left(M_{j}\right)$ is supersolvable. So we have that $\left\langle a, M_{j+1}\right\rangle \leq N_{G}\left(M_{j}\right)$ satisfies Max, and then $M_{j+1}^{a}=M_{j+1}$. This contradicts the assumption on $j$. Thus, $i=j$, and the statement is proved. Therefore, $N_{G}\left(F_{D}\right)=N_{A}\left(F_{D}\right) N_{D}\left(F_{D}\right)=N_{A}\left(F_{D}\right) D$.

Now we prove that $N_{G}\left(F_{D}\right)$ is supersolvable. In fact, we know that

$$
N_{G}\left(F_{D}\right)=N_{A}\left(F_{D}\right) N_{D}\left(F_{D}\right)
$$

holds; by Lemma 2.2, we have

$$
N_{G}\left(F_{D}\right)=N_{A}\left(F_{D}\right) N_{B}\left(F_{D}\right) .
$$

Passing to the quotient, we see that

$$
\frac{N_{G}\left(F_{D}\right)}{F_{D}}=\frac{N_{A}\left(F_{D}\right) F_{D}}{F_{D}} \frac{N_{B}\left(F_{D}\right) F_{D}}{F_{D}}
$$

is a product of supersolvable $\mathcal{N}$-connected subgroups. Since $h\left(F_{D}\right) \geq 1$, we can apply the inductive hypothesis and use Lemma 3.1 to deduce that $N_{G}\left(F_{D}\right)$ is supersolvable.

The last fact we prove is that $N_{G}\left(F_{D}\right)>D$. Actually, we demonstrate a stronger fact, that is, $\left|N_{G}\left(M_{i}\right): M_{i+1}\right|=\infty$ for all $i: 0, \ldots, t-1$. Proceed by induction on $i$. If $i=0$, we know by Corollary 3.4 that $\left|N_{A}\left(M_{0}\right): M_{0}\right|=\infty$. By Lemma 3.2, $\left|N_{G}\left(M_{0}\right): N_{D}\left(M_{0}\right)\right|=\infty$, and then $\left|N_{G}\left(M_{0}\right): M_{1}\right|=\infty$. Suppose that $i \geq 1$. By the inductive hypothesis, we know that

$$
\left|N_{G}\left(M_{i-1}\right): M_{i}\right|=\infty
$$


Keeping in mind that $M_{i}$ is nilpotent, we want to prove that

$$
\left|N_{N_{G}\left(M_{i-1}\right)}\left(M_{i}\right): M_{i}\right|=\infty .
$$

Let $W=N_{G}\left(M_{i-1}\right), F_{W}=\operatorname{Fit}(W)$ and $K=F_{W} \cap F_{D}$. Clearly, $K \leq M_{i}$. If $K=M_{i}$, then by Corollary 3.4, we get the thesis. Otherwise, consider $N_{W}(K)$. By Corollary 3.4, we have that $\left|N_{W}(K): K\right|=\infty$. Since $K \triangleleft N_{D}\left(M_{i-1}\right)$, we have that $N_{W}(K) \geq N_{D}\left(M_{i-1}\right)$. For this reason, the factorization

$$
N_{W}(K)=N_{A}(K) N_{D}\left(M_{i-1}\right)
$$

holds. Consider $R=N_{A}(K) K, S=R_{N_{W}(K)}$ (the core of $R$ in $N_{W}(K)$ ) and $T=S \cap F_{W}$. By construction, $K \leq T \triangleleft N_{W}(K)$. Define $L=T N_{D}\left(M_{i-1}\right)$. Considering the quotient

$$
\frac{L}{K}=\frac{T}{K} \frac{N_{D}\left(M_{i-1}\right)}{K},
$$

we have that $T / K$ is nilpotent, and by Proposition $2.4, N_{D}\left(M_{i-1}\right) / K$ is abelian.

In particular, $L / K$ is supersolvable, and it is the product of two nilpotent $\mathcal{N}$ connected subgroups; hence, by Lemma $3.1, L / K$ is nilpotent. We know that $\left|L / K: M_{i} / K\right|=\infty$, so by Lemma 3.3, we get

$$
\left|N_{L / K}\left(M_{i} / K\right): M_{i} / K\right|=\infty,
$$

which implies

$$
\left|N_{L}\left(M_{i}\right): M_{i}\right|=\infty,
$$

and then

$$
\left|N_{N_{G}\left(M_{i}\right)}\left(M_{i}\right): M_{i}\right|=\infty .
$$

Thus, by Lemma 3.2,

$$
\left|N_{G}\left(M_{i}\right): N_{D}\left(M_{i}\right)\right|=\infty,
$$

and then the thesis $\left|N_{G}\left(M_{i}\right): M_{i+1}\right|=\infty$.

Gathering all these facts, we obtain $N_{G}\left(F_{D}\right) \in \mathscr{B}$ and $N_{G}\left(F_{D}\right)>D$. This fact contradicts the maximality of $D$. So $h(A)=h(A \cap D)$, or in other words, $|G: D|<\infty$. Thus, $D_{G}$ is a supersolvable normal subgroup of $G$. By Theorem 2.6, $G / D_{G}$ is supersolvable, and by Lemma 3.1, $G$ is supersolvable.

When the factors are not supersolvable, the situation is unclear.

Problem 3.6. Let $G$ be a group and $A, B \leq G=A B$, where $A$ and $B$ are polycyclic $\mathcal{N}$-connected subgroups. Is it true that $G$ is a polycyclic group? 


\section{Proof of Theorem B}

We introduce some technical lemmas that are needed in the sequel.

Considering a product of Černikov groups, it is possible to describe the finite residual in terms of the finite residuals of the factors.

Lemma 4.1. Let $G=A B$ be a Černikov group. If we denote by $A_{0}, B_{0}$ and $G_{0}$ the finite residuals of, respectively, $A, B$ and $G$, then $G_{0}=\left\langle A_{0}, B_{0}\right\rangle$.

Proof. By a lemma of Amberg (see for instance [1, Lemma 1.2.5]), the group $\left\langle A_{0}, B_{0}\right\rangle$ has finite index in $G$, and therefore $G_{0} \leq\left\langle A_{0}, B_{0}\right\rangle$. The other inclusion is clear.

Before going further, we prove the following.

Lemma 4.2. Let $G$ be a periodic group and $1 \neq A \leq G$ a divisible abelian subgroup. Let $a \in A$ and $u \in C_{G}(x)$ for all $x \in A$ such that $a \in\langle x\rangle$. Then $u \in C_{G}(A)$.

Proof. Let $h \in A$, and let $|h|=m$. Consider $a_{1} \in A$ such that $a_{1}^{m}=a$. Therefore, $\left(a_{1} h\right)^{m}=a_{1}^{m}=a$. Hence, the following holds:

$$
u \in C_{G}\left(a_{1}\right) \cap C_{G}\left(a_{1} h\right) \leq C_{G}(h),
$$

which is the thesis.

Notation 4.3. Given a group $G$ such that $G=A B$, where $A, B \leq G$, and an element $g \in G$, we set

$$
\Pi_{A}(g)=\{a \in A \mid \text { there exists } b \in B \text { such that } a b=g\},
$$

and for any subset $X$ of $G$, we set

$$
\Pi_{A}(X)=\bigcup_{x \in X} \Pi_{A}(x)
$$

We are now ready to state and prove the main theorem of this section. The proof is divided in two cases to simplify the reading: firstly the case in which we assume that $A$ and $B$ are $p$-groups and then the general case.

Theorem 4.4. Let $G$ be a group, and let $A, B$ be two subgroups of $G$ such that $G=A B$. Assume that $A$ and $B$ are $\mathcal{N}$-connected and Černikov p-groups, where $p$ is a prime number. Then $G$ is a Černikov p-group. 
Proof. Since $A$ and $B$ are Černikov $p$-groups, denote by $A_{0}$ and $B_{0}$ the finite residuals of, respectively, $A$ and $B$. We know that they are both a direct product of a finite number of copies of the Prüfer $p$-group. We divide the proof into 4 steps. In the first three, we assume, in addition, that $A_{0} \cap B_{0}=1$.

Step 1. Consider $a \in A_{0}$ and $b \in B_{0}$, and let $H=\langle a, b\rangle$. We prove that if $C_{G}(H)$ is infinite, then the set $C_{A_{0}}(H) \cup C_{B_{0}}(H)$ is infinite.

We know that $H$ is nilpotent by $\mathcal{N}$-connection. Using the notation introduced above, let $A^{*}=\Pi_{A}\left(C_{G}(H)\right)$ and $B^{*}=\Pi_{B}\left(C_{G}(H)\right)$. Since $C_{G}(H) \subseteq A^{*} B^{*}$, we have that $A^{*} \cup B^{*}$ is infinite. Without loss of generality, we can suppose that $A^{*}$ is infinite. For all $x \in A^{*}$, there exists a $y \in B$ such that $x y \in C_{G}(H)$. In particular, $b^{x y}=b$, from which $b^{x}=b^{y^{-1}} \in b^{B}$, where $b^{B}$ is a conjugacy class in $B$. Since $b \in B_{0}$, we have that $b^{B}$ is finite; thus there exists an infinite subset $X \subseteq A^{*}$ such that $b^{x}=b^{x_{0}}$ for all $x \in X$ and for a fixed $x_{0} \in X$. Consequently, $\left\{x_{0} x^{-1} \mid x \in X\right\} \subseteq C_{A}(b)$ is infinite. Therefore, $C_{A_{0}}(b)$ is infinite too, and since $a \in A_{0}$ is abelian, we can conclude by observing that

$$
C_{A_{0}}(b)=C_{A_{0}}(\langle a, b\rangle)=C_{A_{0}}(H) .
$$

Step 2. Suppose that $Z(G)=1$; we claim that there exist $a \in A_{0}$ and $b \in B_{0}$ such that $C_{B_{0}}(a)=1=C_{A_{0}}(b)$.

In fact, consider the set $\left\{C_{B_{0}}(a) \mid a \in A_{0}\right\}$; it clearly admits a minimal element. Let $C=C_{B_{0}}(a)$ be one such minimal element. If $C \neq 1$, then by divisibility and minimality, we have that, for all $x$ such that $a \in\langle x\rangle$, also $C_{B_{0}}(\langle x\rangle)=C_{B_{0}}(\langle a\rangle)$. Therefore, we can apply Lemma 4.2 and conclude that $C_{B_{0}}(a)=C_{B_{0}}\left(A_{0}\right)$. So $C_{G}(C) \geq\left\langle A_{0}, B_{0}\right\rangle$, and hence it has finite index. Since $G$ is a $p$-group, this implies that $Z(G) \neq 1$. Thus, if $Z(G)=1$, then the minimal element of the set $\left\{C_{B_{0}}(a) \mid a \in A_{0}\right\}$ is exactly $\{1\}$. The same holds for $\left\{C_{A_{0}}(b) \mid b \in B_{0}\right\}$.

Step 3. Now our objective is to prove that $Z(G) \neq 1$.

Suppose that $Z(G)=1$. Then, by step 2, there exist $a \in A_{0}$ and $b \in B_{0}$ such that $C_{B_{0}}(a)=1=C_{A_{0}}(b)$. By step $1, C_{G}(\langle a, b\rangle)$ is finite. Moreover, by $\mathcal{N}$ connection, we can deduce that $1 \neq Z(\langle a, b\rangle) \leq C_{G}(\langle a, b\rangle)$, namely $C_{G}(\langle a, b\rangle)$ is nontrivial and finite. We can choose $a$ and $b$ such that $\left|C_{G}(\langle a, b\rangle)\right|$ is minimal. Let $1 \neq u \in C_{G}(\langle a, b\rangle)$ and $x \in A_{0}$, where $a \in\langle x\rangle$; thus $C_{G}(\langle x, b\rangle) \leq C_{G}(\langle a, b\rangle)$; hence equality holds, and in particular, $u \in C_{G}(\langle x, b\rangle) \leq C_{G}(\langle x\rangle)$. By Lemma 4.2, we have $u \in C_{G}\left(A_{0}\right)$. Analogously, we can show that $u \in C_{G}\left(B_{0}\right)$, and then $C_{G}(u) \geq\left\langle A_{0}, B_{0}\right\rangle$, which implies $Z(G) \neq 1$.

Step 4 . We claim that $G$ is a Černikov group.

First of all, we show that $G$ is hypercentral proving that every nontrivial quotient group of $G$ has nontrivial center. In fact, since all the hypotheses pass to quotient 
groups, it is sufficient to show that $Z(G) \neq 1$. So if $A_{0} \cap B_{0}=1$, then we obtain our thesis by step 3. Otherwise, denoting $K:=A_{0} \cap B_{0} \neq 1$, it is true that $C_{G}(K) \geq\left\langle A_{0}, B_{0}\right\rangle$ has finite index. Hence, as we observed in the previous steps, $Z(G) \neq 1$. In conclusion, since a hypercentral $p$-group with Min-n is a Černikov $p$-group (see [7]), the theorem is proved.

We can now prove Theorem B.

Proof of Theorem B. Since $A$ and $B$ are Černikov groups, we know that the finite residual $A_{0}$ and $B_{0}$ have the following structure:

$$
A_{0}=\underset{i=1}{\stackrel{s}{X}} P_{i}, \quad B_{0}=\underset{j=1}{\stackrel{t}{X}} Q_{j},
$$

where, in $A_{0}$, each $P_{i}$ is a direct product of $h_{i}$ copies of Prüfer $p_{i}$-groups, in which $p_{i}$ are distinct primes for $i: 1, \ldots, s$; analogously, in $B_{0}$, each $Q_{j}$ is the direct product of $k_{j}$ copies of Prüfer $q_{j}$-groups, in which $q_{j}$ are distinct primes for $j: 1, \ldots, t$.

In terms of minimality, this means $h_{i}=m\left(P_{i}\right)$ and $k_{j}=m\left(Q_{j}\right)$. So we have

$$
m(A)+m(B)=m\left(A_{0}\right)+m\left(B_{0}\right)=\sum_{i=1}^{s} m\left(P_{i}\right)+\sum_{j=1}^{t} m\left(Q_{j}\right) .
$$

We proceed by induction on $m(A)+m(B)$. If $m(A)=0$ (or $m(B)=0$ ), then $B$ is a subgroup of finite index in $G$; hence $B_{G}$ is a Černikov normal subgroup of finite index in $G$, but clearly, a virtually Černikov group is a Černikov group, so we have the thesis. Otherwise, we can assume that $m(A) \geq 1$ and $m(B) \geq 1$. For the previous theorem, we can suppose that $A_{0}$ and $B_{0}$ are not both $p$-groups. Hence, without loss of generality, we can also assume that $h_{1}, h_{2} \geq 1$ and $k_{1} \geq 1$. If $p_{i} \neq q_{j}$ for all $i$ and $j$, then $\left\langle A_{0}, B_{0}\right\rangle$ is abelian by $\mathcal{N}$-connection, so we assume $p_{1}=q_{1}$.

Let $P:=P_{1}$. Since $P$ char $A_{0}$, we have that $P \triangleleft A$. Consider $N_{G}(P)$. By the Wielandt lemma (see [10]), we know that the following factorization holds:

$$
N_{G}(P)=N_{A}(P) N_{B}(P)=A N_{B}(P) .
$$

Moreover, we know

$$
Q_{2} \times \cdots \times Q_{t} \leq C_{B}(P) \leq N_{B}(P) .
$$

Consider two cases. 
Case (1) If $Q_{1} \leq N_{B}(P)$, then $\left|G: N_{G}(P)\right|<\infty$, so we can reduce ourselves to proving that $N_{G}(P)$ is Černikov. Now, $P$ is Černikov, and $N_{G}(P) / P$ is Černikov by the inductive hypothesis; hence $N_{G}(P)$ is Černikov.

Case (2) If $Q_{1} \not \leq N_{B}(P)$, denote $B^{*}:=N_{B}(P)$. We have that $B_{0} \cap B^{*}<B_{0}$ and $\left|B^{*}: B_{0} \cap B^{*}\right|<\infty$, so if $B_{0}^{*}$ is the finite residual of $B^{*}$, we deduce that

$$
B_{0}^{*} \leq B_{0} \cap B^{*}<B_{0} .
$$

Hence, $B_{0}^{*}$ and $B_{0}$ are both direct products of a finite number of Prüfer groups and $B_{0}^{*}<B_{0}$, and $B_{0}$ has no proper subgroups of finite index; then this fact allows us to apply the inductive hypothesis on $N_{G}(P)$ and to see that it is Černikov. By Lemma 4.1, we deduce that the finite residual of $N_{G}(P)$ is exactly $\left\langle A_{0}, B_{0}^{*}\right\rangle$, from which we have $\left[P_{i}, Q_{j}\right]=1$ for all $i: 1, \ldots, s$ and for all $j: 2, \ldots, t$. Now, considering $P_{2}$ and repeating the same argument, if case (1) holds, the proof is done; otherwise, $\left[P_{i}, Q_{j}\right]=1$ must hold for all $i: 1, \ldots, s$ and for all $j: 1,3, \ldots, t$. Gathering all these facts, we conclude that $\left[A_{0}, B_{0}\right]=1$, from which $\left\langle A_{0}, B_{0}\right\rangle$ is abelian and it has finite index in $G$.

Moreover, $G$ is the product of two subgroups with Min; thus it satisfies Min-n, i.e. the minimal condition on normal subgroups (see [1]).

Finally, every subgroup of finite index of $G$ satisfies Min-n (see [11]); hence $\left\langle A_{0}, B_{0}\right\rangle$ is abelian Černikov, and then $G$ is Černikov.

Proof of Corollary 1.3. By Proposition 2.5, $A$ and $B$ are Černikov groups, and by Theorem B, $G$ is also Černikov. The solvability of $G$ follows from the fact that if $G_{0}$ is the finite residual of $G$, then $G_{0}$ is abelian and $G / G_{0}$ is solvable by Theorem 2.6.

Acknowledgments. This paper is part of the $\mathrm{PhD}$ thesis of the author, which was supervised by Carlo Casolo whose contribution has been fundamental. Most sincere thanks are also due to Orazio Puglisi and to the thesis referees Bernhard Amberg and Francesco de Giovanni for their accurate comments and suggestions.

\section{Bibliography}

[1] B. Amberg, Factorisation of infinite groups, Habilitationschrift, Universität Mainz, 1973.

[2] B. Amberg, S. Franciosi and F. de Giovanni, Products of Groups, Oxford Math. Monogr., The Clarendon, New York, 1992.

[3] A. Carocca, A note on the product of $\mathscr{F}$-subgroups in a finite group, Proc. Edinburgh Math. Soc. (2) 39 (1996), no. 1, 37-42. 
[4] N. Itô, Über das Produkt von zwei abelschen Gruppen, Math. Z. 62 (1955), 400-401.

[5] O. H. Kegel, Produkte nilpotenter Gruppen, Arch. Math. (Basel) 12 (1961), 90-93.

[6] J. C. Lennox and D. J. S. Robinson, The Theory of Infinite Soluble Groups, Oxford Math. Monogr., Oxford Science, Oxford, 2004.

[7] D. H. McLain, Remarks on the upper central series of a group, Proc. Glasgow Math. Assoc. 3 (1956), 38-44.

[8] D. J. S. Robinson, A Course in the Theory of Groups, 2nd ed., Grad. Texts in Math. 80, Springer, New York, 1996.

[9] D. Segal, Polycyclic Groups, Cambridge Tracts in Math. 82, Cambridge University, Cambridge, 1983.

[10] H. Wielandt, Über Produkte von nilpotenten Gruppen, Illinois J. Math. 2 (1958), 611-618.

[11] J. S. Wilson, Some properties of groups inherited by normal subgroups of finite index, Math. Z. 114 (1970), 19-21.

Received September 1, 2020; revised November 27, 2020.

\section{Author information}

Corresponding author:

Giulio Francalanci, Dipartimento di Matematica e Informatica "U. Dini”, Università degli Studi di Firenze, Viale Morgagni 67/A, Firenze, Italy.

E-mail: giulio.francalanci@unifi.it 